\title{
EFFECTIVENESS OF SURGICAL RECONSTRUCTION TO RESTORE RADIOCARPAL JOINT MECHANICS AFTER SCAPHOLUNATE LIGAMENT INJURY. AN IN VIVO MODELING STUDY
}

\author{
Joshua E. Johnson ${ }^{\mathrm{a}}$, Phil Lee ${ }^{\mathrm{b}}$, Terence E Mclff ${ }^{\mathrm{c}}$, E. Bruce Tobyc, and Kenneth J. \\ Fischer ${ }^{\mathrm{a}, \mathrm{c},{ }^{*}}$ \\ aDepartment of Mechanical Engineering, University of Kansas, 3138 Learned Hall, Lawrence, \\ Kansas, 66045, USA \\ bHoglund Brain Imaging Center, University of Kansas Medical Center, 3901 Rainbow Boulevard, \\ Kansas City, Kansas, 66160, USA \\ 'Department of Orthopedic Surgery, University of Kansas Medical Center, 3901 Rainbow \\ Boulevard, Kansas City, Kansas, 66160, USA
}

\section{Abstract}

\begin{abstract}
Disruption of the scapholunate ligament can cause a loss of normal scapholunate mechanics and eventually lead to osteoarthritis. Surgical reconstruction attempts to restore scapholunate relationship and shows improvement in functional outcomes, but postoperative effectiveness in restoring normal radiocarpal mechanics still remains a question. The objective of this study was to investigate the benefits of surgical repair by observing changes in contact mechanics on the cartilage surface before and after surgical treatment. Six patients with unilateral scapholunate dissociation were enrolled in the study, and displacement driven magnetic resonance image basedsurface contact modeling was used to investigate normal, injured and postoperative radiocarpal mechanics. Model geometry was acquired from images of wrists taken in a relaxed position. Kinematics were acquired from image registration between the relaxed images, and images taken during functional loading. Results showed a trend for increase in radiocarpal contact parameters with injury. Peak and mean contact pressures significantly decreased after surgery in the radiolunate articulation and there were no significant differences between normal and postoperative wrists. Results indicated surgical repair improves contact mechanics after injury and that contact mechanics can be surgically restored to be similar to normal. This study provides novel contact mechanics data on the effects of surgical repair after scapholunate ligament injury. With further work, it may be possible to more effectively differentiate between treatments and degenerative changes based on in vivo contact mechanics data.
\end{abstract}

\footnotetext{
(C) 2013 Elsevier Ltd. All rights reserved.

"Corresponding author, 1530 W. 15th St, 3138 Learned Hall, Lawrence, Kansas, 66045, USA, Phone: +1 785864 2994, Fax: +1 785 864 5254, fischer@ku.edu.

Publisher's Disclaimer: This is a PDF file of an unedited manuscript that has been accepted for publication. As a service to our customers we are providing this early version of the manuscript. The manuscript will undergo copyediting, typesetting, and review of the resulting proof before it is published in its final citable form. Please note that during the production process errorsmaybe discovered which could affect the content, and all legal disclaimers that apply to the journal pertain.

Conflict of interest statement

The authors do not have any conflicts of interest to disclose.
} 


\section{Keywords}

Scapholunate Dissociation; Modeling; Contact Pressure; Magnetic Resonance Imaging; Posttraumatic Osteoarthritis

\section{Introduction}

Wrist joint motion involves a complex interplay between articulating geometries and ligamentous constraints, which play a vital role in joint stability (Berger, 1997). The scapholunate interosseous ligament (SLIL) is the primary stabilizer of the scapholunate (SL) joint (Kijima and Viegas, 2009). Disruption of the SLIL is a common injury, known as scapholunate dissociation (Kuo and Wolfe, 2008), and can lead to instability. Scapholunate instability disrupts the normal scapholunate relationship (Ruby et al., 1987). The misalignment results in abnormal radiocarpal kinematics and load transfer (Ruby et al., 1987; Sokolow, 2001). Subsequent changes in distributions of articular surface contact are associated with progressive degeneration known as scapholunate advanced collapse (SLAC), the most common cause of wrist osteoarthritis (Watson and Brenner, 1985).

Osteoarthritis (OA) is the most functionally and economically debilitating of the degenerative joint diseases (Buckwalter et al., 2004). Both age and joint injuries are known risk factors for OA (Anderson et al., 2011). Cartilage damage can occur from direct injury to the joint, or from progressive instability due to ligamentous injuries. There is a high risk of OA after ligament injuries (Anderson et al., 2011). SLIL injury, if untreated, generally leads to radiocarpal OA (Roberts et al., 2006).

Due to its subtlety, scapholunate dissociation is not often diagnosed early. Also, the rate of progression to SLAC arthropathy varies between individuals. Thus, time from SLIL injury that allows for successful repair is not established (Pomerance, 2006), and ideal treatment when the SLIL is irreparable is unknown (Pollock et al., 2010). The various treatments have inconsistent postoperative outcomes (Saffar et al., 1999), so more data on treatment methods may improve outcomes.

Contact pressure and changes in contact locations and patterns are important mechanical factors in the pathomechanics of OA (Andriacchi et al., 2004). Contact mechanics can be evaluated efficiently by surface contact modeling based on magnetic resonance image (MRI). Joint mechanics data from surface contact modeling can illustrate injury contact patterns and show effectiveness of surgical interventions.

Cadaveric studies cannot adequately assess complex in vivo conditions (active muscle forces and dynamic stability), nor the effectiveness of surgery after healing or at other future times. Cadaveric studies that have investigated treatments for scapholunate dissociation, have primarily examined limited fusions (Viegas and Patterson, 1997). Few studies have investigated in vivo wrist joint mechanics, though recent work in our lab has shown the effects of SLIL injury on radiocarpal mechanics. There does not appear to be any prior work on efficacy of surgical repair to restore normal radiocarpal joint mechanics after SLIL injury.

Hence, the goal of this study was to investigate immediate benefits of surgical repair by observing changes in contact mechanics on the cartilage surface before and after surgical treatment. Our hypothesis was that peak and mean contact pressures would increase with injury, and decrease postoperatively to values near normal. 


\section{Materials and methods}

Six human subjects were enrolled for protocols approved by the internal review board. Subjects were males with a mean age of 31.3 years (22 to 55). Subjects were diagnosed with unilateral scapholunate dissociation, without visual indication of OA (no focal defects or bone-on- bone contact observed on MRI, and arthroscopic evaluation in some cases), by a board certified physician (injured wrists). Contralateral wrists, with no prior history of injury, were used as controls (normal wrists). Injured wrists were re-evaluated 12 to 15 weeks after surgical repair (postoperative wrists).

Functional data was collected for normal, injured and postoperative wrists. Grip strength levels were measured using a hand dynamometer (Baseline $\left.{ }^{\circledR}\right)$. Pain levels were measured using a visual analogue scale $(0=$ minimum to $10=$ maximum $)$ for four conditions: during rest, during daily activities (with splint), during grip strength test and after grip strength test.

MRI was acquired using constructive interference steady state (CISS) or dual echo steady state (DESS) sequences in a 3T clinical scanner. Two image sets were acquired for each wrist. Injured wrists were first imaged at high resolution (CISS: in-plane voxel $0.15 \times 0.15$ $\mathrm{mm}$, scan time $\sim 12$ mins; DESS: in-plane voxel $0.22 \times 0.2 \mathrm{~mm}$, scan time 4.5 mins; out-ofplane thickness $0.5 \mathrm{~mm}$ for both) with the hand relaxed (unloaded). Then, images were acquired during functional grasp (loaded) at reduced resolution (CISS: in-plane voxel $0.30 \times 0.30 \mathrm{~mm}$, scan time $196 \mathrm{~s}$; DESS: in-plane voxel $0.31 \times 0.31 \mathrm{~mm}$, scan time $188 \mathrm{~s}$; outof-plane thickness $1.0 \mathrm{~mm}$ for both) to minimize scan time (Fig. 1). Grasp involved constantly gripping to a specified target $(3.125 \mathrm{psi})$ for the duration of the scan. This target was previously determined to create minimal discomfort while gripping for up to four minutes. Reduced scan times were to minimize subject fatigue and motion artifacts (especially with injury), while acquiring images with adequate resolution. Wrists were braced to ensure consistent loaded positions during grasp (Fig. 2). The normal and postoperative wrists were similarly scanned.

Surface contact modeling required model geometries, kinematics and a contact relationship (i.e. contact pressure linearly proportional to strain). Radius, lunate and scaphoid bones with their cartilage surfaces, were segmented from the high resolution images (ScanIP,

Simpleware, Exeter, UK). These were wrapped with triangular surface elements to create undeformed 3D surface models of the radiolunate and radioscaphoid articulations for the normal, injured and postoperative wrists (Fig. 3).

Kinematics were acquired using markerless volume-based image registration between relaxed and functionally loaded image sets (Analyze 5.0, Analyze Direct, Overland Park, KS). Bone only segmentations were used for image registration based on the assumption that bones deformed negligibly under light grasp, while cartilage deformed even under the low loading conditions. First, the radius was used as a fixed reference to align the loaded and unloaded image sets. The transformation from this step was used to place the loaded images of carpal bones in the unloaded image coordinate system. Lastly, unloaded carpal bone image sets were registered to the "transformed" loaded carpal bone image sets. Thus, scaphoid and lunate kinematics were obtained for the normal, injured and postoperative wrists. The kinematics placed the unloaded carpal bone models in the functionally loaded state.

Model geometries and kinematics were implement in the Joint_Model program to obtain displacement constrained contact mechanics (Kwak et al., 2000). Contact areas and locations of contact were determined directly from overlapping surface elements of the rigid bodies in the functionally loaded state. A uniform thickness of $1 \mathrm{~mm}$ was assigned to each articular surface based on typical values (Johnson et al., 2012). While the cartilage thickness 
was uniform, articular surfaces were the actual anatomical surfaces from the MR images. The overlap and total thickness were used to estimate deformation at each node, which gave a first order approximation of the local cartilage strain. Since the images were acquired over a relatively long period of time, it was assumed that the cartilage was substantially relaxed. Hence, an effective compressive relaxation modulus of $4 \mathrm{MPa}$ was used as material property (Kwak et al., 2000). Local contact pressure was determined from the nodal strain and material property. The nodal contact pressures were integrated over the contact area associated with each node, to obtain contact force. The ratio of contact force to contact area gave the mean contact pressure.

We also determined contact areas directly from the loaded MR images. Regions of articular surface contact were visible in the images. These were segmented individually using bsplines from each image in the image set, to obtain arcs of contact. Each contact arc length was calculated from the b-splines and multiplied by the slice thickness. These were summed to obtain the effective contact area for each articulation. These "direct" contact areas were compared to the model contact areas to verify modeling accuracy. Locations of scaphoid and lunate centroids of contact were also determined from the loaded images to obtain scapholunate intercentroid distances, which were compared between the normal, injured and postoperative wrists.

Contact pressure distributions were qualitatively compared between normal, injured and postoperative wrists. Grip strength, peak and mean contact pressures, contact forces, model contact areas and intercentroid distances, were compared between the three conditions using oneway repeated measures analysis of variance (ANOVA). Two-way ANOVA was used to compare pain levels between state and condition, and also contact areas between method (model, direct) and condition. All data were tested for parametric assumption. Tukey's LSD was used for post hoc analysis with significance defined as $p<0.05$. Linear regression analysis was performed to determine the relationship between model and direct contact areas. Power analysis was also performed to estimate number of subjects that would be required to observe significant differences.

\section{Results}

All data is presented as means ( \pm standard error). For all subjects, locations of scaphoid and lunate contact on the radius were commonly in the mid to dorsal region and also along the entire fossa (volar to dorsal). With injury, scaphoid contact appeared to shift dorsal and sometimes also laterally toward the styloid region, while lunate contact shifted away from dorsal towards the middle. Figure 4 shows the dorsal-lateral shift in radioscaphoid contact with injury (B) compared to normal (A). Surgery appeared to return the contacts somewhat towards their normal locations (Fig. 4C). There were no significant differences in intercentroid distances between the normal, injured and postoperative wrists (15.06 \pm 0.69 , $16.59 \pm 0.78$ and $16.76 \pm 1.05 \mathrm{~mm}$, respectively).

Grip strength significantly decreased in the injured wrists $(66.8 \pm 19.2 \mathrm{lbs})$ compared to the normal $(110.2 \pm 9.8 \mathrm{lbs})$. Grip strength was also significantly lower in the postoperative wrists $(74.2 \pm 13.1 \mathrm{lbs})$ compared to the normal but there were no significant differences between the injured and postoperative wrists. Pain levels were significantly higher when performing daily activities $(2.0 \pm 0.5)$, compared to when at rest $(0.9 \pm 0.5)$. There was a strong trend for increase in pain levels during the grip strength test $(1.7 \pm 0.6)$, compared to when at rest. There were no significant differences in pain levels between when at rest and after the grip strength test $(1.3 \pm 0.5)$. Also, pain levels were significantly higher in the injured wrists $(3.6 \pm 1.3)$ compared to normal $(0)$. However, pain levels were similar to normal, postoperatively $(0.8 \pm 0.4)$. 
In the radiolunate articulation, there was a trend for increase in peak and mean contact pressures with injury (Figs. 5, 6). There was a significant decrease in peak $(p=0.023)$ and mean $(\mathrm{p}=0.044)$ contact pressures postoperatively, compared to the injured wrists (Figs. 5, 6). There were no significant differences between the normal and postoperative wrists. In the radioscaphoid joint, no significant differences were observed between normal, injured and postoperative wrists (Figs. 5, 6), though there was a strong trend for decrease in peak contact pressure postoperatively, compared to the injured wrists.

Contact force showed a trend for increase in the injured wrists for both the radiolunate and radioscaphoid articulations (Table 1). After surgery, contact force showed a strong trend for decrease but there were no significant differences in contact force (Table 1).

Similarly, model contact area showed a trend for increase with injury, and decrease after surgery in both the radiolunate and radioscaphoid articulations (Table 1). There were no significant differences between contact areas in both articulations. Also, there were no significant differences between model and direct contact areas in both articulations. Model contact areas were within $10 \mathrm{~mm}^{2}$ of the direct contact areas in the radiolunate articulation (Table 1). Aside from the postoperative wrists, model contact areas were within $2 \mathrm{~mm}^{2}$ of the direct contact areas in the radioscaphoid articulation (Table 1). Regression analysis of all contact area data, showed a significant relationship between model and direct contact area measurements $\left(\mathrm{R}^{2}=0.88\right.$; slope $=0.79$ and $\mathrm{R}^{2}=0.78$; slope $=1.3$ for the radiolunate and radioscaphoid articulations respectively, $\mathrm{p}<0.001$ ).

To differentiate between effects of surgical repair on contact parameters after injury, results from power analysis indicated that typically 7 to 10 subjects will be required. To observe changes in normal mechanics after injury, 10 to 15 subjects would be needed.

\section{Discussion}

We investigated the in vivo effects of SLIL injury on normal radiocarpal mechanics, and subsequent surgical repair to restore normal scapholunate relationship, using MRI-based surface contact modeling. An adverse mechanical response to the injury was observed from the tendency of contact parameters to increase post injury. The study showed a significant decrease in contact pressures in the radiolunate joint of the postoperative wrists, compared to the injured, and there were no significant differences between normal and postoperative wrists. A trend toward decrease in other contact parameters was also observed post surgery. The results supported our hypothesis that surgical intervention lowers elevated contact parameters after injury, returning them to near normal.

It is believed that SLIL disruption alone may not produce significant widening of the SL interval (Kuo and Wolfe, 2008). SL diastasis visible on plain radiographs, may also indicate progressive damage to the secondary stabilizers (Manuel and Moran, 2010). SL diastasis after injury, was generally observed by a separation in the locations of scaphoid and lunate contact on the radius articular surface qualitatively (Fig. 4), and also from the trend toward increase in intercentroid distance quantitatively. Normal intercentroid distances from this study $(15.06 \pm 0.69 \mathrm{~mm})$ were similar to those observed in previous studies $(14.91 \mathrm{~mm})$ (Patterson and Viegas, 1995).

SL diastasis visible in the unloaded position (Fig. 3 center), suggests progressive instability from damage to the secondary stabilizers. The dorsal (scaphoid) and volar (lunate) shifts in contact locations observed after injury are indicative of dorsal intercalated segment instability deformity, where the lunate extends and the scaphoid collapses into flexion.(Kuo and Wolfe, 2008) The radiocarpal articulations are considered incongruous (Patterson and Viegas, 1995), hence the non-uniform/unsymmetrical contact patterns were expected. 
Functional data were consistent with physical examinations of SLIL injury. There is a loss of grip strength and increase in pain levels after injury (Manuel and Moran, 2010), as observed in this study, likely due to the shift in contact locations and abnormal load transfer through the joint. Surgical repair attempts to minimize pain and improve function. Pains levels were similar to normal postoperatively, which is a common outcome of surgery (Kuo and Wolfe, 2008; Manuel and Moran, 2010). No significant improvements in grip strength after surgery could be a result of subjects being cautious and protecting the repair. However, improvements in grip strength have also been observed over time (Chabas et al., 2008; Garcia-Elias et al., 2006).

Peak and mean contact pressures were expected to increase with injury in both radiolunate and radioscaphoid articulations. Prior work in our lab has shown peak and mean contact pressures to significantly increase in the radioscaphoid articulation, and peak pressure to significantly increase in the radiolunate articulation. An increase in contact pressure above a certain threshold, may be a risk factor for initiation of degenerative changes. However, a more critical mechanism may be a shift in contact location (particularly in the radioscaphoid articulation) to normally unloaded articular regions, even though increase in contact pressure is not significant.

While there were no significant differences, contact forces and model contact areas in particular, showed a trend for increase with injury in both articulations. Prior work in our lab has also shown all contact parameters to increase significantly with injury in the radioscaphoid articulation. This corresponds to the clinically observed SLAC pattern, where degenerative changes are initially observed at the radioscaphoid region, and then progress medially (Weissand Rodner, 2007). The grasp activity was always the same, so the trend toward increase in contact forces with injury may be due to co-contracture, to stabilize the injured joint. This is also indicated by the fact that after surgery, contact forces showed a strong trend to decrease to near normal values postoperatively. Experiments have shown normal contact forces and contact areas to be higher in the radioscaphoid articulation compared to the radiolunate articulation (Patterson and Viegas, 1995), which was observed in this study.

The significant relationship between model and direct contact area measurements from regression analysis (and no significant differences from two-way ANOVA), suggests reliability of the MRI-based surface contact modeling technique. The usefulness of the method of estimating contact area from grasp MR images to verify in vivo modeling accuracy, has been previously shown (Johnson et al., 2012). Due to the possibility of motion artifacts with human subjects (especially for loaded scans), the consistently higher values may indicate a tendency to overestimate direct contact area measurements.

The observed trends and variation in data could be attributed to the differences in injury/ instability patterns between the subjects. SL instability can be clinically classified into predynamic, dynamic, static and SLAC stages (Manuel and Moran, 2010; Walsh, 2002). Of the six subjects, two were acute (< four weeks from injury and diagnosis), where the instability may have been minimal. One was subacute (between four weeks and six months). Three were chronic (> six months), which may have been sufficient time for progressive instability to develop.

Differences in stages of instability (Manuel and Moran, 2010; Walsh, 2002), variations in manifestation/progression of instability (Watson and Brenner, 1985; Werner et al., 2007), difficulty in treatment selection (Chabas et al., 2008; Garcia-Elias et al., 2006) and inconsistent outcomes (Chabas et al., 2008; Fortin and Louis, 1993; Pollock et al., 2010; Pomerance, 2006; Saffar et al., 1999), all highlight the importance of tools to evaluate soft 
tissue injuries and corrective measures, and the risk of OA development (Anderson et al., 2011). MRI-based surface contact modeling can be a useful tool to determine joint contact mechanics.

One limitation of the study was modeling a single pose to evaluate SLIL injury and surgical repair. Scapholunate motion occurs predominantly in flexion-extension (Gardner et al., 2006), and corresponding changes in kinematics after injury may be better visualized during this motion. However, there is minimal scapholunate motion during most functional activities (Gardner et al., 2006), and these positions may better indicate changes in mechanics with instability. The light grasp pose corresponded to the power grip activity, which the wrist frequently undergoes during activities of daily living. Also, the clenched fist anteroposterior (longitudinal compressive load) view is commonly used to diagnose SL instability in its early stages (Manuel and Moran, 2010). Hence, the light grasp pose was considered appropriate to investigate the effects of injury and surgical repair, since subsequent mechanics have functional implications. It was also easier to acquire MR images to obtain model geometry and boundary conditions using a quasi-static pose. In the future, investigating mechanical changes during other dynamic functional activities, such as dart thrower's motion or circumduction, may also provide additional useful data.

To simplify the analysis, uniform cartilage thickness was assumed for each surface, which may have led to variation in strain estimates. This may also explain some of the variation in contact pressure results. Assigning spatially varying cartilage thickness values may further improve accuracy of acquiring subject-specific contact data. Also, the relatively low number of subjects may have resulted in fewer significant differences in contact parameters. Power analysis indicated that observed trends may become significant by doubling the number of subjects.

The surgeries performed were four direct ligament repairs, one three-ligament tenodesis (a modified Brunelli procedure according to Garcia-Elias et al. (2006)), and one scaphocapitate arthrodesis. Significant decreases in radiolunate peak and mean contact pressure postoperatively, indicated the benefits of having surgery. Surgical outcomes appeared positive from similarities in these radiolunate parameters between normal and postoperative wrists. However, the benefits may be limited by the severity of instability at the time of diagnosis. The longer the time between injury and treatment, the greater the possibility of the instability worsening. As mentioned before, with increasing severity, instability can progress to the periscaphoid region and eventually towards the lunate side. Decrease in postoperative contact pressures in the radiolunate articulation may indicate the effectiveness of treatments to correct less severe instabilities, which may not have had sufficient time to fully develop. No significant decrease in postoperative contact pressures suggests a more severe deformity in the radioscaphoid articulation, which may not have been completely corrected after surgery, indicating that more robust reconstruction techniques may be required for effective outcomes. Surgery tended to reduce the SL diastasis (Fig. 3 right), however not all contact locations appeared completely restored (Fig. 4). Lack of significant reduction of the scapholunate interval may also suggest that longer time may be needed to heal after repair. This may provide an explanation as to why degenerative changes are sometimes observed even after surgical intervention. Due to the relatively low number of subjects, it was not possible to address effectiveness of reconstruction by technique. The mechanical outcomes of surgeries are expected to be different for ligament repairs, tendon weaves and salvage procedures, and an overall comparison of repair to injured and normal, though appearing positive, may not accurately present the complete picture. Further work is warranted to categorize reconstructive surgeries according to their biomechanical outcomes. 
In conclusion, this study provides novel radiocarpal contact mechanics data on the efficacy of surgical repair after SLIL injury that further strengthens the importance of surgery to manage/halt the progression of instability and minimize/eliminate the risk of developing OA. Future work would include pooling subjects according to type of surgery and also investigating subsequent benefits at 1-2 years post surgery. The potential is to provide insight into the effectiveness of surgery from a contact mechanics perspective and better establish the extent to which each surgical repair/reconstruction restores normal joint mechanics.

\section{Acknowledgments}

We would like to acknowledge NIH R01EB008709 grant funding, Columbia University for Joint_Model software, A. Schmitt for technical assistance with MR imaging and M. Humphrey, E.Tobaben, M. Mandala, S. Modaresi and Q. Zheng for assistance with image processing.

\section{References}

Anderson DD, Chubinskaya S, Guilak F, Martin JA, Oegema TR, Olson SA, Buckwalter JA. Posttraumatic osteoarthritis: Improved understanding and opportunities for early intervention. Journal of Orthopaedic Research. 2011; 29:802-809. [PubMed: 21520254]

Andriacchi TP, Mündermann A, Smith RL, Alexander EJ, Dyrby CO, Koo S. A Framework for the in Vivo Pathomechanics of Osteoarthritis at the Knee. Annals of Biomedical Engineering. 2004; 32:447-457. [PubMed: 15095819]

Berger RA. The ligaments of the wrist. A current overview of anatomy with considerations of their potential functions. Hand Clinics. 1997; 13:63-82. [PubMed: 9048184]

Buckwalter JA, Saltzman C, Brown T. The impact of osteoarthritis: implications for research. Clinical Orthopaedics and Related Research. 2004:S6-S15. [PubMed: 15480076]

Chabas J-F, Gay A, Valenti D, Guinard D, Legre R. Results of the Modified Brunelli Tenodesis for Treatment of Scapholunate Instability: A Retrospective Study of 19 Patients. Journal of Hand Surgery. 2008; 33:1469-1477. American Volume. [PubMed: 18984325]

Crisco JJ, Pike S, Hulsizer-Galvin DL, Akelman E, Weiss AP C, Wolfe SW. Carpal bone postures and motions are abnormal in both wrists of patients with unilateral scapholunate interosseous ligament tears. Journal of Hand Surgery. 2003; 28:926-937. American Volume. [PubMed: 14642507]

del Piñal F, Studer A, Thams C, Glasberg A. An All-Inside Technique for Arthroscopic Suturing of the Volar Scapholunate Ligament. Journal of Hand Surgery. 2011 American Volume (in press).

Fortin PT, Louis DS. Long-term follow-up of scaphoid-trapezium-trapezoid arthrodesis. Journal of Hand Surgery. 1993; 18:675-681. American Volume. [PubMed: 8349980]

Garcia-Elias M, Lluch AL, Stanley JK. Three-Ligament Tenodesis for the Treatment of Scapholunate Dissociation: Indications and Surgical Technique. Journal of Hand Surgery. 2006; 31:125-134. American Volume. [PubMed: 16443117]

Gardner MJ, Crisco JJ, Wolfe SW. Carpal kinematics. Hand Clinics. 2006; 22:413-420. [PubMed: 17097463]

Johnson JE, McIff TE, Lee P, Toby EB, Fischer KJ. Validation of radiocarpal joint contact models based on images from a clinical MRI scanner. Computer Methods in Biomechanics and Biomedical Engineering. 2012

Kijima Y, Viegas SF. Wrist anatomy and biomechanics. Journal of Hand Surgery. 2009; 34:15551563. American Volume. [PubMed: 19801111]

Kuo CE, Wolfe SW. Scapholunate Instability: Current Concepts in Diagnosis and Management. Journal of Hand Surgery. 2008; 33:998-1013. American Volume. [PubMed: 18656780]

Kwak SD, Blankevoort L, Ateshian GA. A Mathematical Formulation for 3D Quasi- Static Multibody Models of Diarthrodial Joints. Computer Methods in Biomechanics and Biomedical Engineering. 2000; 3:41-64. [PubMed: 11264838]

Manuel J, Moran SL. The diagnosis and treatment of scapholunate instability. Hand Clinics. 2010; 26:129-144. [PubMed: 20006251] 
Patterson R, Viegas SF. Biomechanics of the wrist. Journal of Hand Therapy. 1995; 8:97-105. [PubMed: 7550635]

Pollock PJ, Sieg RN, Baechler MF, Scher D, Zimmerman NB, Dubin NH. Radiographic Evaluation of the Modified Brunelli Technique Versus the Blatt Capsulodesis for Scapholunate Dissociation in a Cadaver Model. Journal of Hand Surgery. 2010; 35:1589-1598. American Volume. [PubMed: 20888495]

Pomerance J. Outcome After Repair of the Scapholunate Interosseous Ligament and Dorsal Capsulodesis for Dynamic Scapholunate Instability Due to Trauma. Journal of Hand Surgery. 2006; 31:1380-1386. American Volume. [PubMed: 17027803]

Roberts C, Porter M, Wines AP, Shadbolt B. The association of scapho-trapeziotrapezoid osteoarthrosis and scapholunate dissociation. Hand Surgery. 2006; 11:135-141. [PubMed: 17405194]

Ruby LK, An KN, Linscheid RL, Cooney WP 3rd, Chao EY. The effect of scapholunate ligament section on scapholunate motion. Journal of Hand Surgery. 1987; 12:767-771. American Volume. [PubMed: 3655239]

Saffar P, Sokolow C, Duclos L. Soft tissue stabilization in the management of chronic scapholunate instability without osteoarthritis. A 15-year series. Acta Orthopaedica Belgica. 1999; 65:424-433. [PubMed: 10675937]

Sokolow C. Anatomy and histology of the scapholunate ligament. Hand Clinics. 2001; 17:77. [PubMed: 11280161]

Viegas SF, Patterson RM. Load mechanics of the wrist. Hand Clinics. 1997; 13:109-128. [PubMed: 9048187]

Walsh JJ. Current status of scapholunate interosseous ligament injuries. Journal of the American Academy of Orthopaedic Surgeons. 2002; 10:32. [PubMed: 11809049]

Watson HK, Brenner LH. Degenerative disorders of the wrist. Journal of Hand Surgery. 1985; 10:1002-1006. American Volume. [PubMed: 4078281]

Weiss KE, Rodner CM. Osteoarthritis of the Wrist. Journal of Hand Surgery. 2007; 32:725-746. American Volume. [PubMed: 17482013]

Werner FW, Short WH, Green JK, Evans PJ, Walker JA. Severity of scapholunate instability is related to joint anatomy and congruency. Journal of Hand Surgery. 2007; 32:55-60. American Volume. [PubMed: 17218176] 


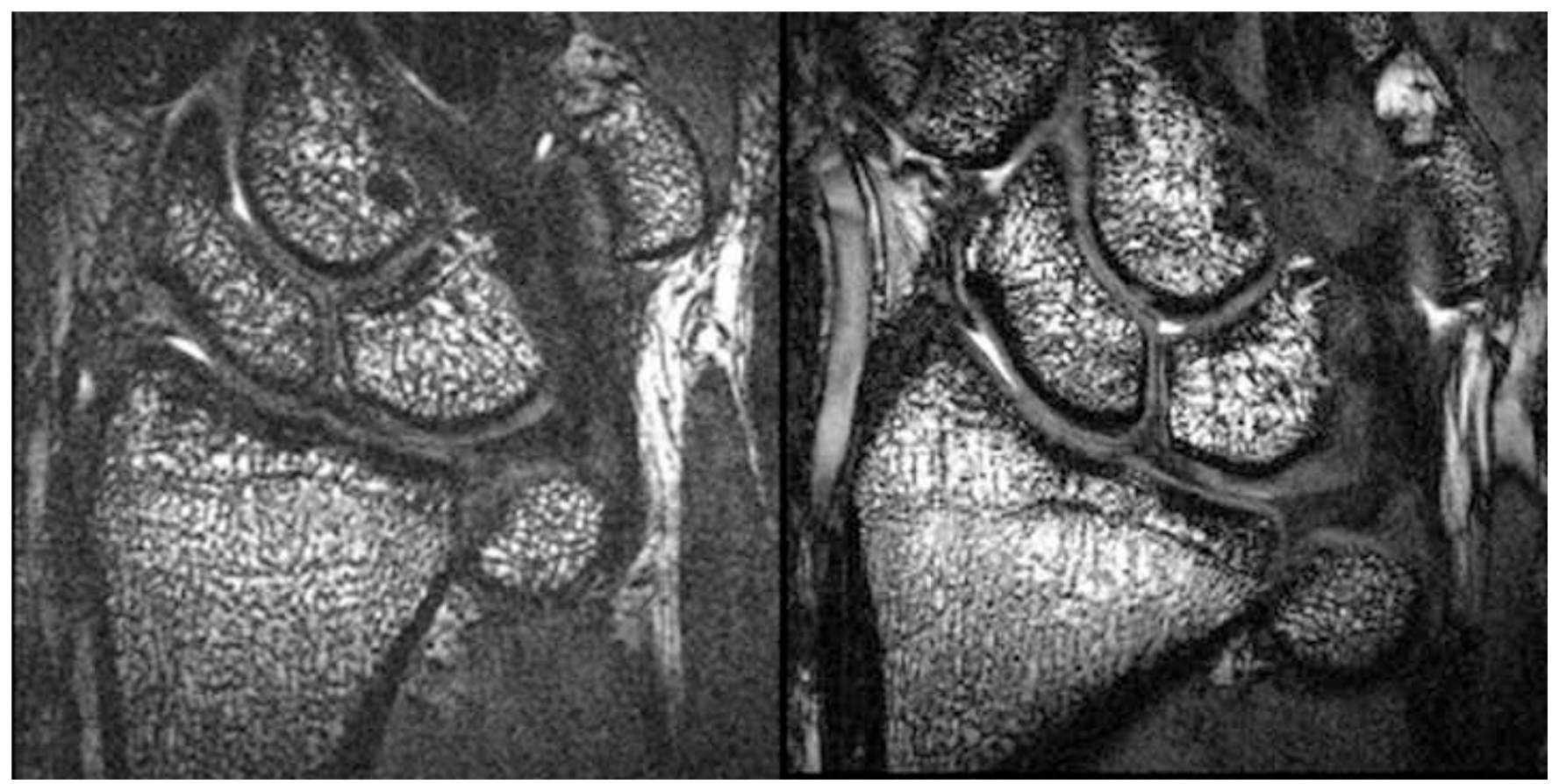

Figure 1.

Sample MRI images of the normal wrist of Subject 1. Left shows a high resolution slice of the unloaded wrist used for model construction, while right shows a lower resolution slice acquired during functional loading for image registration. 


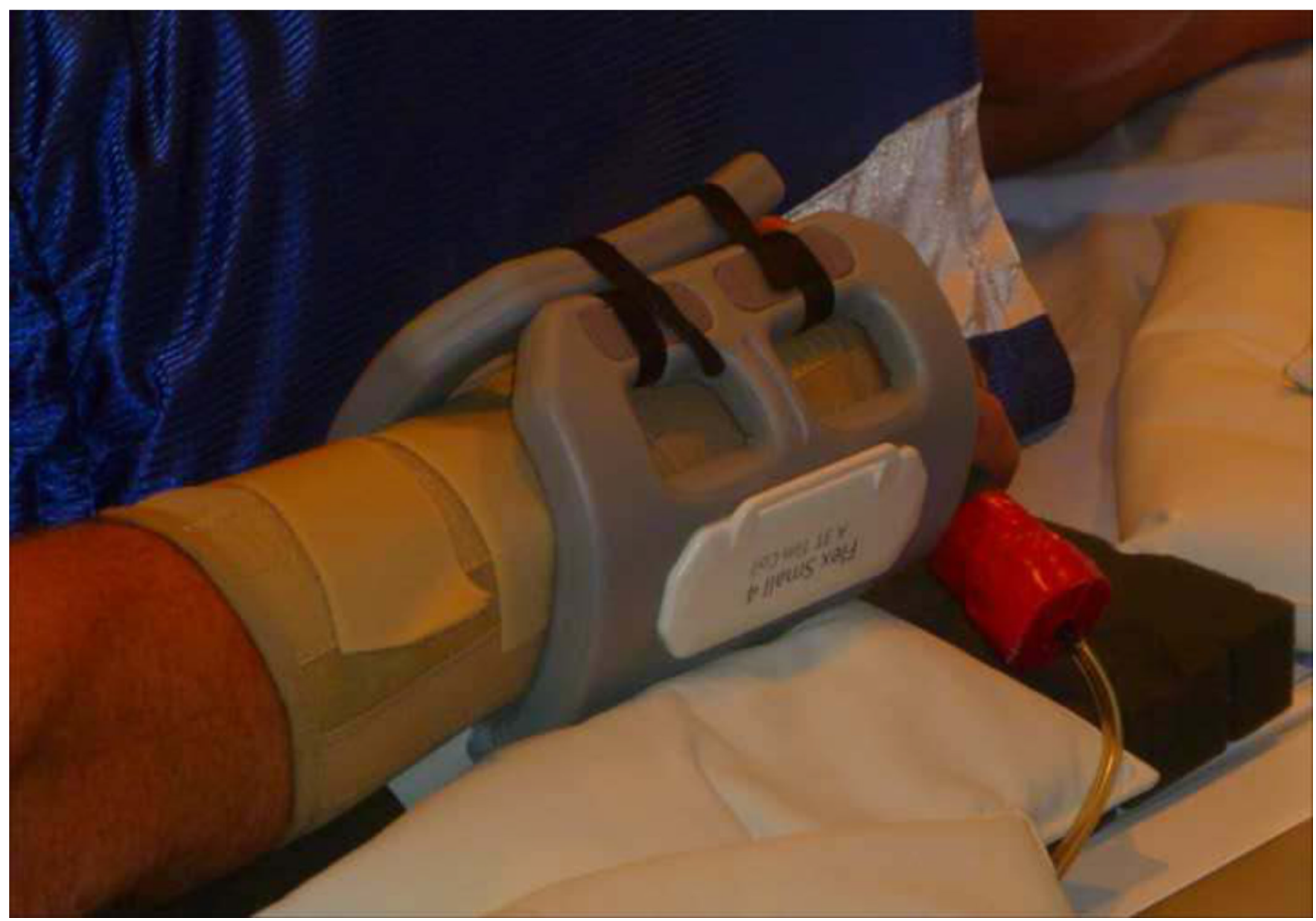

Figure 2.

Example wrist of a subject with the grip device in the active grasp position. The wrist was also braced for consistent loaded positions. 


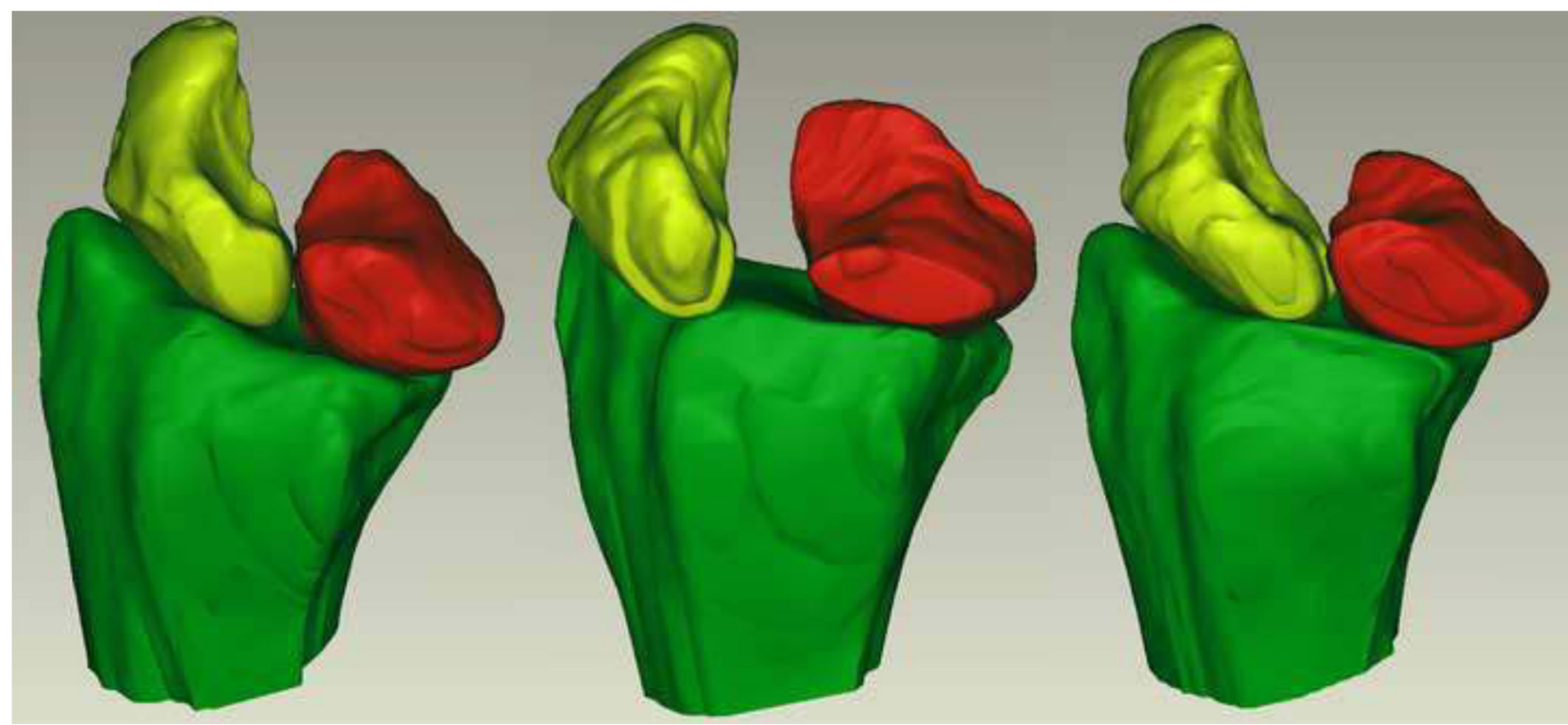

Figure 3.

Normal (left), injured (center) and postoperative (right) surface models of the radiocarpal joint in the unloaded position, for Subject 5, from a dorsal/posterior view. Radius, lunate and scaphoid bones are colored green, red and yellow respectively. 


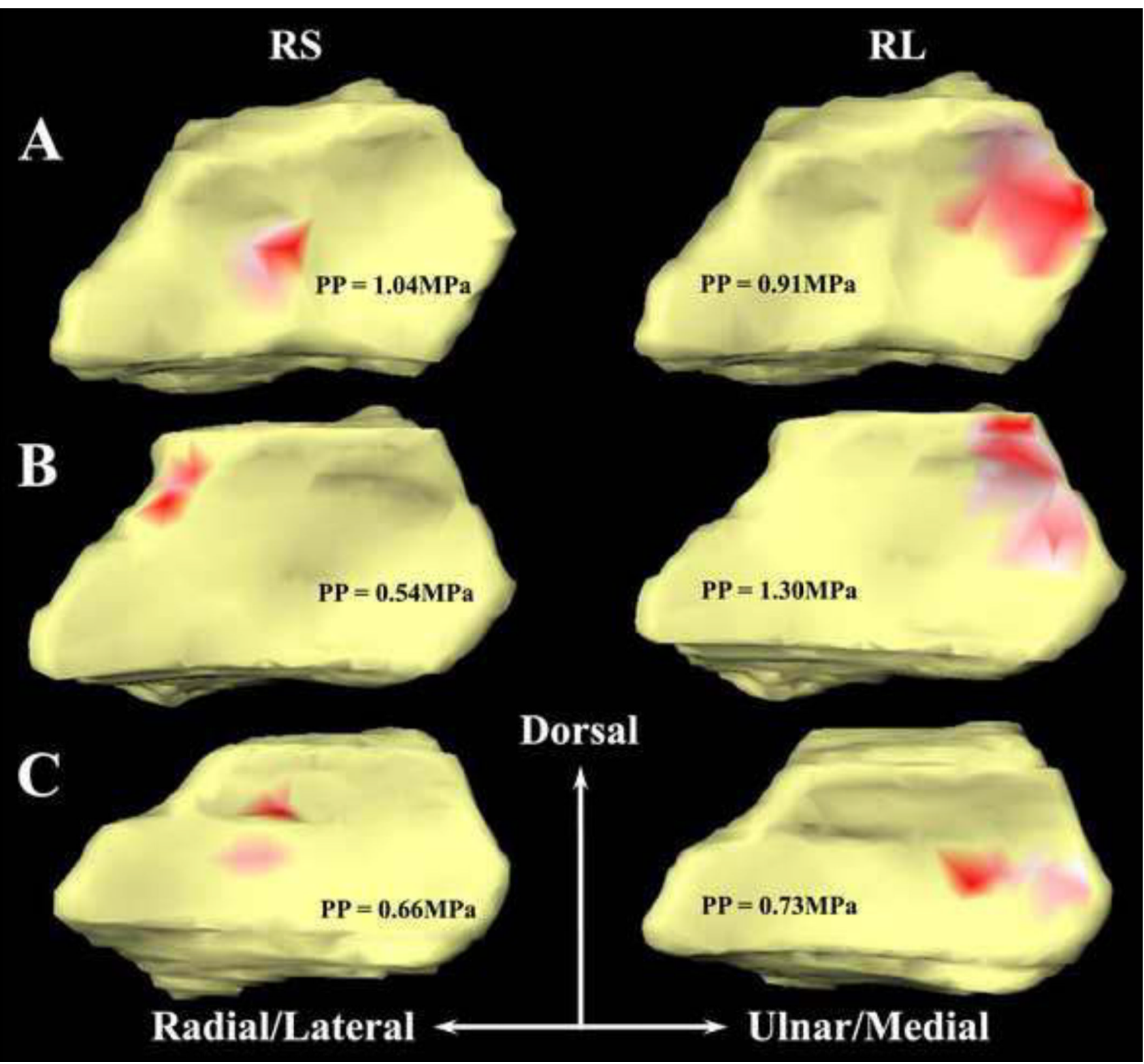

Figure 4.

Normal (A), injured (B) and postoperative (C) contact locations of Subject 5, for radioscaphoid $(\mathrm{RS})$ and radiolunate $(\mathrm{RL})$ articulations, shown on the radius cartilage. Magnitude of contact pressures vary linearly from white (minimum) to dark red (maximum) for each articulation. Peak pressure (PP) values are also shown. The images for this particular subject illustrate clear separation of the scaphoid and lunate in the injured wrist, primarily due to scaphoid motion. After repair, the scaphoid moves medially to a position approaching the normal contact location. While this grossly illustrates the typical overall behavior, not all subjects exhibited these contact patterns. 


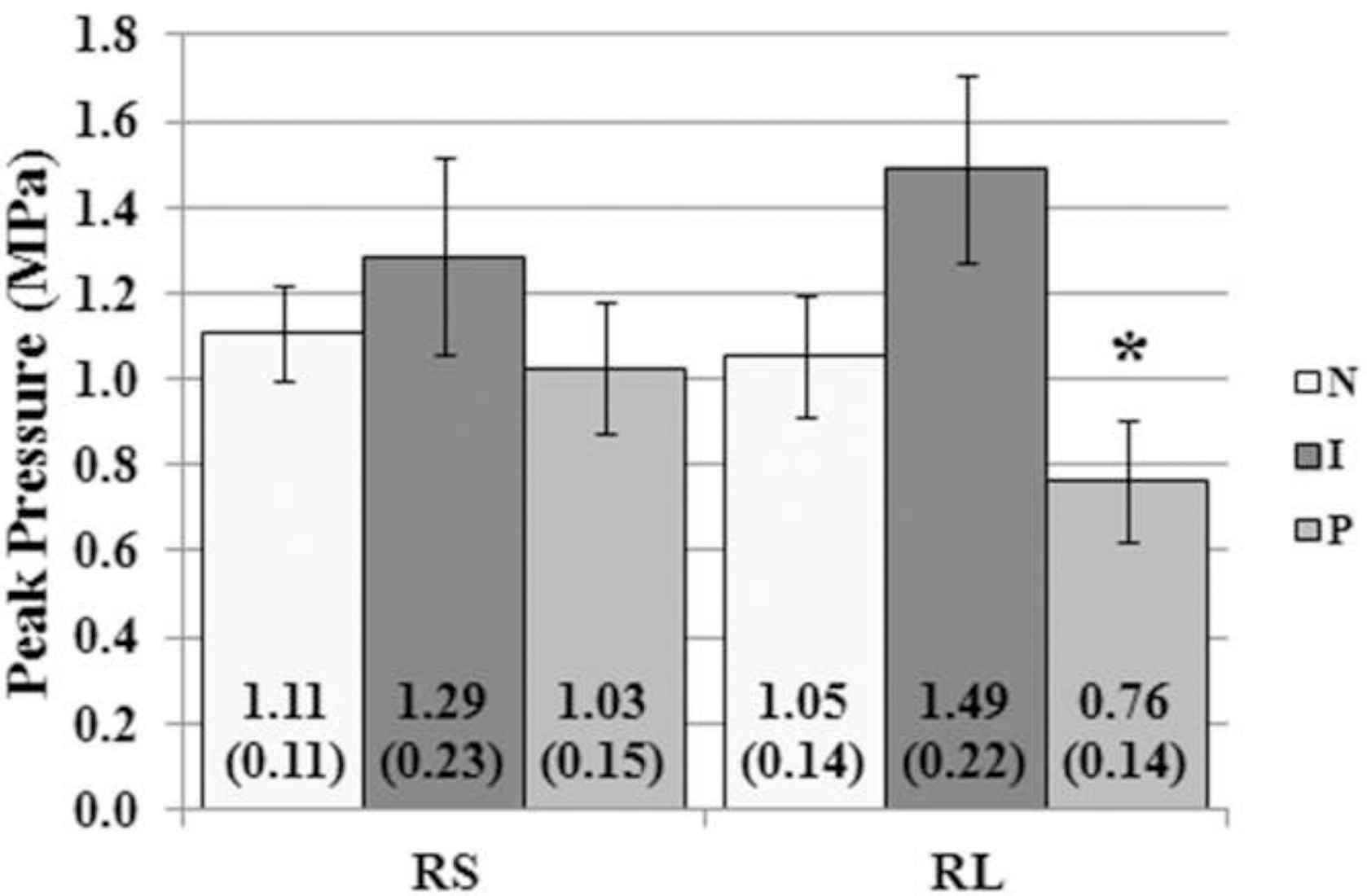

Figure 5.

Means ( \pm standard errors) of radioscaphoid (RS) and radiolunate (RL) peak contact pressures for normal $(\mathrm{N})$, injured $(\mathrm{I})$ and postoperative $(\mathrm{P})$ wrists. * indicates significant difference from injured. 


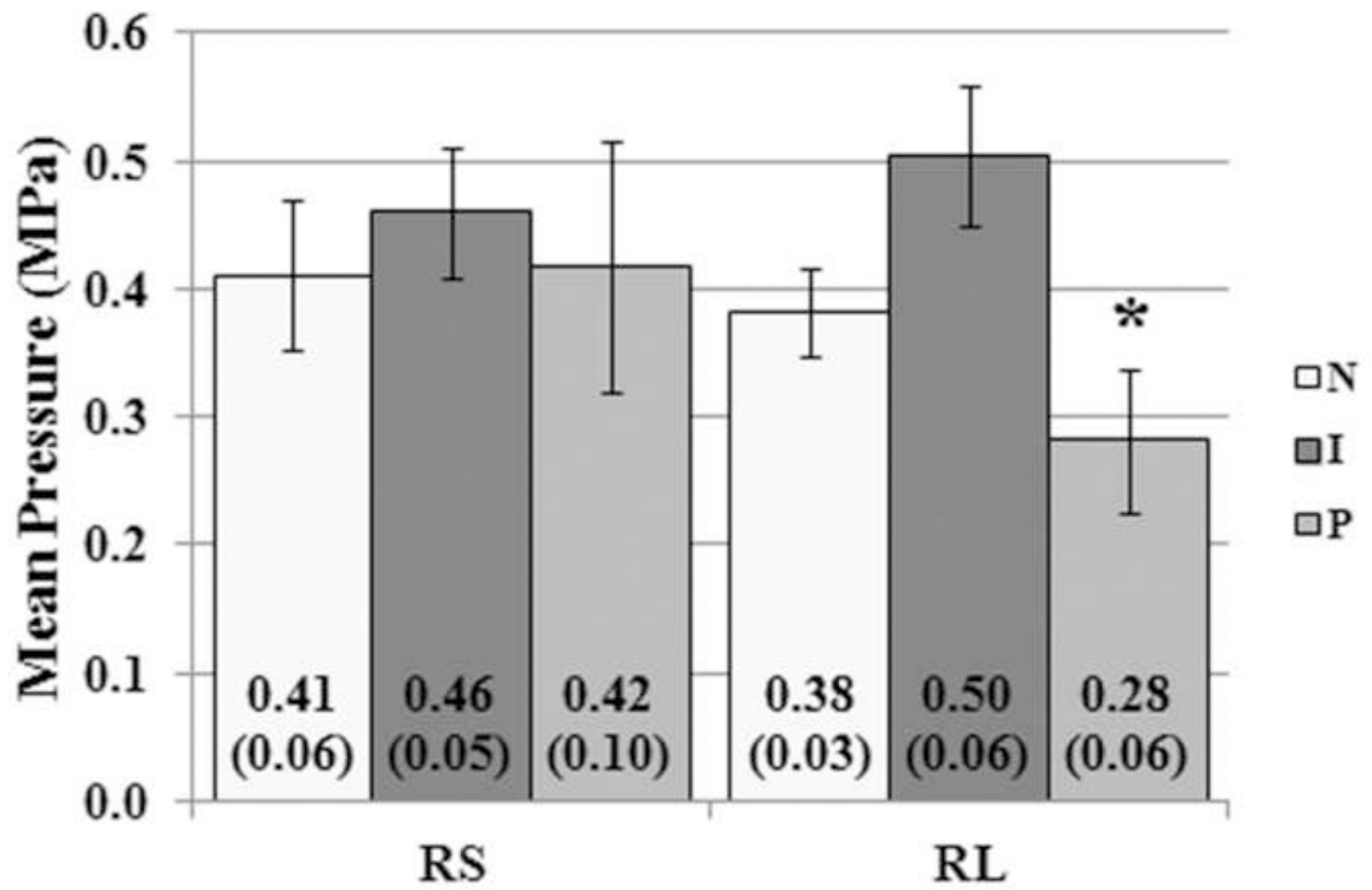

Figure 6.

Means ( \pm standard errors) of radioscaphoid (RS) and radiolunate (RL) mean contact pressures for normal $(\mathrm{N})$, injured $(\mathrm{I})$ and postoperative $(\mathrm{P})$ wrists. * indicates significant difference from injured. 
Table 1

Contact force, model contact area and direct contact area data for normal, injured and postoperative wrists for both radiolunate $(\mathrm{RL})$ and radioscaphoid $(\mathrm{RS})$ articulations

\begin{tabular}{|c|c|c|c|c|}
\hline \multicolumn{2}{|c|}{ Mean ( \pm Standard Error) } & \multirow{2}{*}{$\frac{\text { Normal }}{23(6)}$} & \multirow{2}{*}{$\begin{array}{c}\text { Injured } \\
37(6)\end{array}$} & \multirow{2}{*}{$\frac{\text { Postoperative }}{14(5)}$} \\
\hline \multirow{3}{*}{$\mathbf{R L}$} & Contact Force $(N)$ & & & \\
\hline & Model Contact Area $\left(\mathrm{mm}^{2}\right)$ & $58(14)$ & $70(9)$ & $46(6)$ \\
\hline & Direct Contact Area $\left(\mathrm{mm}^{2}\right)$ & $61(15)$ & $79(13)$ & $50(6)$ \\
\hline \multirow{3}{*}{ RS } & Contact Force (N) & $27(6)$ & $40(12)$ & $22(6)$ \\
\hline & Model Contact Area $\left(\mathrm{mm}^{2}\right)$ & $65(8)$ & $82(23)$ & $59(14)$ \\
\hline & Direct Contact Area $\left(\mathrm{mm}^{2}\right)$ & $64(8)$ & $81(16)$ & $77(8)$ \\
\hline
\end{tabular}

